\title{
TWO-DIMENSIONAL AXISYMMETRIC WINDING MODEL FOR FINITE DEFORMATION
}

\author{
K. ÄRÖLÄ ${ }^{1}, R$. VON HERTZEN ${ }^{2}$ \\ ${ }^{1}$ VTT - Technical Research Centre of Finland \\ Smart Machines, Structural Dynamics \\ P.O.Box 1000, FI-02044 VTT, Finland \\ e-mail: kilwa.arola@vtt.fi \\ ${ }^{2}$ Lappeenranta University of Technology \\ Laboratory of Fatigue and Strength \\ P.O.Box 20, FIN-53851 Lappeenranta, Finland \\ e-mail: raimo.von.hertzen@lut.fi
}

\begin{abstract}
A two-dimensional axisymmetric winding model for wound rolls of thin web is developed. The model accounts for radial and axial displacements and radial, circumferential, axial and shear stresses. The roll build-up is modeled as an incremental accretion process. The material behaviour of the roll is considered as hyperelastic, orthotropic and radially nonlinear. The numerical solution is developed using the finite element method and the total Lagrangian formulation. The model is applied to the winding of paper rolls. It is shown that centrifugal forces may considerably affect the resulting stress distributions. For nonzero Poisson's ratios significant edge effects in the roll stresses are found. In particular, high shear stresses and shear stress gradients are discovered in the vicinity of the core near the roll ends. A remarkable stress leveling phenomenon is found where the effect of a non-constant incoming web tension is evened out in the roll axial direction.
\end{abstract}

\section{Introduction}

Thin sheet media such as paper, magnetic tape, polymer, and metal are generally wound into compact rolls for the subsequent handling and transportation. The roll form is by far the most economical and practical form of material storage. During the production process, the tensioned thin sheet or web is wrapped on a solid central core. At this stage, the stresses and strains in the roll are built up in an incremental manner. The resulting stresses determine to a large extent the quality of the roll, and provide the most important piece of information for the evaluation of the future durability and functionality of the finished roll. Also, as the trend in winding technology is towards thinner media and faster winding speeds [1], the mechanical stability of the rolls becomes more and more critical. Although experimental data through the sandwiched pull strip method and some other methods can be obtained in laboratory conditions, practical non-destructive techniques to measure the 
internal state of stress of a roll on a production line are still lacking. This applies to finished rolls as well as to rolls still in a wind up. Thus, to evaluate the quality of the winding, there is an obvious need for a physical model of the winding process.

There are several roll defects which may result from nonoptimal stress distributions in a roll. Lack of tension may cause cinching, telescoping and spoking while excessive stresses can lead to increased creep, interlayer buckling, core collapse and starring $[2,3,4,5,6]$. It is known, for example, that desirable radial stresses are large enough to prevent individual layers from slipping, but not too large, to avoid surface damage. However, since the stress build up in a winding roll is a complex process, a reliable roll model is needed to quantify the contribution of various factors influencing the roll quality. In this way design criteria for the roll's state of stress can be developed.

Although winding is a continuous process, it has generally been modeled as an incremental process where successive pretensioned circular hoops are shrunk-fit onto the underlying roll. The motivation for the axisymmetry stems from the fact that any dependence on the azimuth angle is averaged out due to the rolling nip which makes hundreds or thousands of revolutions during a wind up. Thus, the roll is assumed to be a collection of single concentric hoops stuck together. This is an example of an accretion problem of solid mechanics or of the mechanics of growing bodies. Typical other examples are a solidifying body, a growing crystal, a growing solid surface due to spray deposition etc. All such bodies consist of mass elements that became part of the growing body at different times and at different initial stresses. It is typical of growing bodies that a configuration in which the elements of the body would be connected in a stress free state does not exists. This implies that the strain tensor does not satisfy the conventional compatibility condition. It can be shown, however, that the compatibility condition is satisfied when expressed in terms of strain increments or strain rates.

There is an extensive literature on the mechanics of wound rolls. The developed models can be used for the calculation of internal stress and small deformation distributions of wound rolls. Most of them are one-dimensional accounting for the radial change of the stress and displacement fields only. The one-dimensional models can be grouped into four categories according to the constitutive law of the modeled roll. These constitutive laws have been linearly $[7,8,9]$ or nonlinearly $[10,11,12]$ elastic and linearly $[13,14,15]$ or nonlinearly [16] viscoelastic and have treated the material as being orthotropic. The roll has been considered in a plane stress or plane strain state corresponding to very short or long rolls, respectively (magnetic tape packs or large paper rolls, for example), in which uniform mechanical properties along the roll's axial direction are assumed.

Recently, a few models treating the roll as a two-dimensional system have been published $[4,5,6,17]$. In these models, the core and web regions are considered to be of finite width. The stresses and strains are allowed to vary both in the roll's radial and axial directions, and four stress components - radial, circumferential, axial, and shear - as well as two displacements - radial and axial - are included. With these models, nonuniform winding tension, web thickness, elastic moduli, and core stiffness, depending on the axial coordinate, can be treated. In these works, the model has been applied to magnetic tape packs. Zabaras and Liu [4] have examined the effect of the nonuniform winding tension on the stresses in the tape pack. Lee and Wickert $[5,6]$ have treated several realistic cartridge hub (core) designs, and the roles of hub compliance and wound-in tension gradients in setting the tape pack's stress field are considered. Lately, Li and Cao have presented a hybrid approach for the winding process of thin-sheet coils [17]. They develop an approximate multi-layer finite element model to study the coil deformation under gravitational loading. They use in their finite element model the stresses calculated by an incremental winding 
model as initial stresses for the subsequent analysis of the "soft coil" problem and other two-dimensional phenomena. It should be noted, however, that their winding model itself is one-dimensional.

In many winding applications the displacements can be large and the displacement gradients are not small compared to unity. This applies particularly in paper winding, where the radial strains of the paper rolls take typically values of the order $5-10 \%$, and even $15 \%$ in the case of certain soft paper boards. Therefore, the infinitesimal strain theory, utilized usually for strains well below $1 \%$, is not a good approximation for such rolls. Also, as the winding speeds continuously increase due to larger production demands, centrifugal forces may have a significant effect on the resulting stress distributions. Although Benson [12] has treated large deformations in winding and Olsen [18] the effect of centrifugal forces, their models have been one-dimensional. A unified treatment of large deformations and centrifugal forces in a two-dimensional winding model has not yet been presented.

In this work, a two-dimensional winding model for predicting the stress and strain fields within a wound roll of web material, in which the radial, circumferential, transverse, and shear stresses, and the radial and axial strains can vary in both the roll's radial and axial (cross-web) directions, is presented. The material behaviour of the roll is considered as orthotropically anisotropic, linearly elastic in the circumferential and axial directions and nonlinearly elastic in the radial direction. It should be noted that the elastic properties of the bulk roll, and the nonlinear radial modulus in particular, may be quite different from those of a single isolated layer, and are strongly affected by air entrainment in the roll, asperity compliance at the individual web surfaces, ambient air temperature and humidity, and other factors. The roll is built up in an incremental manner and total equilibrium in the roll is required after adding of each single hoop. Here the tensioned hoop is allowed to shrink on the surface of the underlying roll, so that the wound-in tension loss [19] is automatically accounted for. The effect of centrifugal forces is also taken into account. The numerical solution is developed using the finite element method. In particular, finite strains are incorporated in the model using the total Lagrangian formulation. Also the core is modeled by the finite element method so that cores with nonlinear material behaviour can be treated. The model is applied for the analysis of paper rolls. The effect of the finite width of the roll on the stresses inside the roll and near the roll's free ends is studied. Also, the effect of a nonuniform distribution of the incoming winding tension in the roll axial direction is discussed.

The paper proceeds as follows. In Sections 2 and 3 the governing equations of the roll model, and the finite element equations utilizing the total Lagrangian formulation for finite strains, are developed. In Section 4 the linearization and solution of the nonlinear set of equations is demonstrated. The formulation of the constitutive equations of a paper roll are considered in Section 5. The outline of the simulated winding procedure and the reference configuration are discussed in Section 6. In Section 7 numerical results for several winding examples are presented. Finally, the conclusions are drawn in Section 8.

\section{Axisymmetrical kinematics}

In this work the kinematics of the roll is described by using the reference description, in which the independent variable is the position $\mathbf{X}$ of a particle or material point in a reference configuration. Let $\mathbf{e}_{R}, \mathbf{e}_{\Theta}$ and $\mathbf{e}_{Z}$ be the unit base vectors in the cylindrical coordinate system of Fig. 1. The position of a material point in the undeformed reference configuration is expressed as

$$
\mathbf{X}=R \mathbf{e}_{R}+Z \mathbf{e}_{Z}
$$




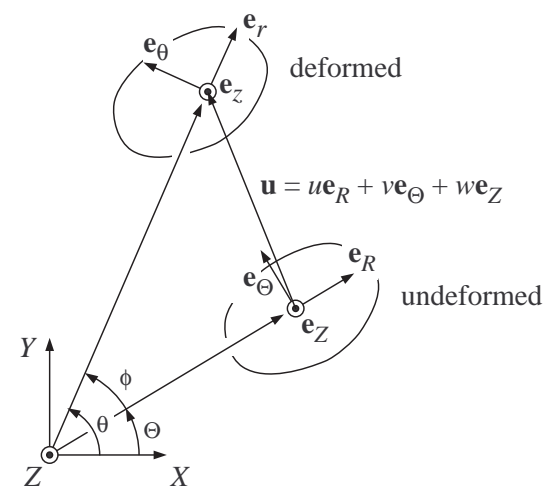

Figure 1: Base vectors e and displacement $\mathbf{u}$ in cylindrical coordinates. The view is projected on a plane of constant $Z$.

where $R$ and $Z$ are the cylindrical coordinates of the material point. The displacement of a particle in terms of the cylindrical unit base vectors may be written as (see Fig. 1)

$$
\mathbf{u}=u \mathbf{e}_{R}+v \mathbf{e}_{\Theta}+w \mathbf{e}_{Z},
$$

where $u=u(R, Z), v \equiv 0$ and $w=w(R, Z)$ due to the axisymmetry of the deformed roll. The position of a point in the current (deformed) configuration is

$$
\mathbf{x}=\mathbf{X}+\mathbf{u} .
$$

The deformation gradient

$$
\mathbf{F}=\frac{\partial \mathbf{x}}{\partial \mathbf{X}},
$$

expressed in cylindrical coordinates in the case of axisymmetric deformation, can be written in matrix form as

$$
[\mathbf{F}]=\left[\begin{array}{ccc}
1+\partial u / \partial R & \partial u / \partial Z & 0 \\
\partial w / \partial R & 1+\partial w / \partial Z & 0 \\
0 & 0 & 1+u / R
\end{array}\right]
$$

Using the deformation gradient, the Green (or Green-Lagrange) strain tensor is written as

$$
\mathbf{E}=\frac{1}{2}\left(\mathbf{F}^{T} \cdot \mathbf{F}-\mathbf{I}\right)
$$

However, due to the limited numerical accuracy of digital computers, the Green strain can be calculated more accurately using the displacement gradient $\mathbf{H}=\mathbf{F}-\mathbf{I}$ leading to the expression

$$
\mathbf{E}=\frac{1}{2}\left(\mathbf{H}+\mathbf{H}^{T}+\mathbf{H}^{T} \cdot \mathbf{H}\right) .
$$

\section{Discretized equilibrium equations}

In this work the problem solution is developed in terms of the total Lagrangian formulation, in which the Lagrangian measures of stress and strain are used and all derivatives and integrals are taken with respect to the material coordinates. The starting point for the 
derivation of the discretized equilibrium equations is the principle of virtual power, which in the total Lagrangian formulation takes the form

$$
\delta P=\int_{\Omega_{0}} \mathbf{S}: \delta \dot{\mathbf{E}} \mathrm{d} \Omega_{0}-\int_{\Omega_{0}} \rho_{0} \mathbf{b} \cdot \delta \mathbf{v} \mathrm{d} \Omega_{0}-\int_{\Gamma_{0}} \mathbf{t} \cdot \delta \mathbf{v} \mathrm{d} \Gamma_{0}=0,
$$

where $\mathbf{S}$ is the second Piola-Kirchhoff stress tensor, $\mathbf{b}$ the body force per unit mass, $\mathbf{t}$ the surface traction, $\delta \dot{\mathbf{E}}$ the virtual rate of Green strain and $\delta \mathbf{v}$ the virtual velocity. The integrations are carried out over the volume $\Omega_{0}$ and boundary $\Gamma_{0}$ of the system. Note that the virtual power equation can be written in several forms using different conjugate pairs of stress and strain. Here, the use of the rate of Green strain and the second PiolaKirchhoff stress is motivated by the simple form of equation (8) and by the choice of the constitutive equation to be used. In the present work, the consitutive model has to take into account the high anisotropy of paper. Hyperelastic materials, in which the stress is found by differentiating a strain-energy function $\psi$ with respect to the strain,

$$
\mathbf{S}=\frac{\partial \psi(\mathbf{E})}{\partial \mathbf{E}},
$$

provide a natural framework for the frame-invariant formulation of an anisotropic material response by simply embodying the anisotropy in the potential $\psi$.

Proceeding in the standard manner and expressing the virtual velocity field in terms of the element shape functions and nodal velocites, and taking into account the arbitrariness of the virtual velocities, the discretized equation of equilibrium for node $I$ emerges as

$$
\mathbf{f}_{I}^{\text {int }}-\mathbf{f}_{I}^{e x t}=\mathbf{0},
$$

where the internal and external forces are

$$
\mathbf{f}_{I}^{i n t}=\int_{\Omega_{0}} \mathbf{B}_{I}^{T}\{\mathbf{S}\} \mathrm{d} \Omega_{0}
$$

and

$$
\mathbf{f}_{I}^{e x t}=\int_{\Omega_{0}} N_{I} \rho_{0} \mathbf{b} \mathrm{d} \Omega_{0}+\int_{\Gamma_{0}} N_{I} \mathbf{t} \mathrm{d} \Gamma_{0},
$$

respectively, and $\{\cdot\}$ denotes Voigt notation. The matrix $\mathbf{B}_{I}$ in the expression of the internal forces relates the rate of Green strain to the nodal velocities. In the axisymmetric case this relation within an element is written as

$$
\{\dot{\mathbf{E}}\}=\sum_{I=1}^{n} \mathbf{B}_{I} \mathbf{v}_{I}
$$

or

$$
\left\{\begin{array}{c}
\dot{E}_{11} \\
\dot{E}_{22} \\
\dot{E}_{33} \\
2 \dot{E}_{12}
\end{array}\right\}=\sum_{I=1}^{n}\left[\begin{array}{ll}
B_{11}^{I} & B_{12}^{I} \\
B_{21}^{I} & B_{22}^{I} \\
B_{31}^{I} & B_{32}^{I} \\
B_{41}^{I} & B_{42}^{I}
\end{array}\right]\left\{\begin{array}{c}
\dot{u}^{I} \\
\dot{w}^{I}
\end{array}\right\}
$$

where $n$ is the number of nodes in the element.

Any quantity $\phi(t)$ within an element is interpolated using the nodal values $\phi_{I}(t)$ and the element shape functions $N_{I}(\boldsymbol{\xi})$ as

$$
\phi(t, \boldsymbol{\xi})=\sum_{I=1}^{n} N_{I}(\boldsymbol{\xi}) \phi_{I}(t) \equiv N_{I}(\boldsymbol{\xi}) \phi_{I}(t)
$$


where $t$ is time, $\boldsymbol{\xi}$ contains the parent element coordinates and $n$ is the number of nodes in the element. We have adopted the conventions that capital indices, $I, J$, refer to node numbers, lower case indices, $i, j, k$, refer to tensor or matrix components and repeated indices mean summation. The derivatives of the shape functions with respect to the undeformed coordinates $\mathbf{X}$ (here with components $R$ and $Z$ ) are needed in the development of the finite element equations. These are obtained from

$$
\frac{\partial N_{I}(\boldsymbol{\xi})}{\partial \mathbf{X}}=\left(\frac{\partial \mathbf{X}}{\partial \boldsymbol{\xi}}\right)^{-T} \frac{\partial N_{I}}{\partial \boldsymbol{\xi}} ; \quad \frac{\partial \mathbf{X}}{\partial \boldsymbol{\xi}}=\sum_{I=1}^{n} \mathbf{X}_{I} \otimes \frac{\partial N_{I}}{\partial \boldsymbol{\xi}}
$$

To get expressions for the components of the matrix B in Eq. (13), the rate of Green stain is first obtained by differentiating Eq. (6) with respect to time. This gives

$$
\dot{\mathbf{E}}=\frac{1}{2}\left(\dot{\mathbf{F}}^{T} \cdot \mathbf{F}+\mathbf{F}^{T} \cdot \dot{\mathbf{F}}\right), \quad \dot{E}_{i j}=\frac{1}{2}\left(\dot{F}_{k i} F_{k j}+F_{k i} \dot{F}_{k j}\right) .
$$

In the axisymmetric case the nonzero components of the rate of Green strain are $\dot{E}_{11}$, $\dot{E}_{22}, \dot{E}_{33}$ and $\dot{E}_{12}\left(=\dot{E}_{21}\right)$, where 1,2 and 3 refer to the radial, axial and circumferential direction, respectively. Using the nodal displacements and shape functions in Eq. (5), the time derivative of the deformation gradient within an element becomes

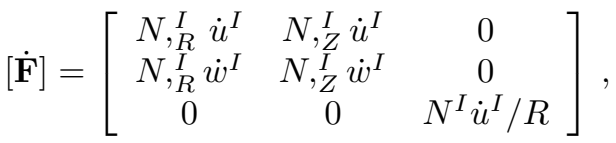

where the notations $\partial(\cdot) / \partial R=(\cdot),_{R}$ and $\partial(\cdot) / \partial Z=(\cdot){ }_{Z}$ have been used. By inserting this interpolated rate of deformation gradient into Eq.(17) and by comparing with Eq. (13), one obtains for the matrix $\mathbf{B}^{I}$ corresponding to node $I$ the expression

$$
\mathbf{B}_{I}=\left[\begin{array}{cc}
F_{11} N,_{R}^{I} & F_{21} N,_{R}^{I} \\
F_{12} N,_{Z}^{I} & F_{22} N,_{Z}^{I} \\
F_{33} N^{I} / R & 0 \\
F_{12} N,_{R}^{I}+F_{11} N,_{Z}^{I} & F_{22} N,_{R}^{I}+F_{21} N,_{Z}^{I}
\end{array}\right] .
$$

By joining the contributions of all nodes $I=1, \ldots, n$ into the matrix $\mathbf{B}=\left[\mathbf{B}^{1}, \ldots, \mathbf{B}^{n}\right]$ and vector $\dot{\mathbf{u}}=\left\{\dot{u}^{1}, \dot{w}^{1}, \ldots, \dot{u}^{n}, \dot{w}^{n}\right\}^{T}$, Eq. (13) can be also written in the form $\{\dot{\mathbf{E}}\}=\mathbf{B} \dot{\mathbf{u}}$.

\section{Linearization and solution}

The global discretized equilibrium equation, obtained by writing Eq. (10) for all degrees of freedom of the model, is

$$
\mathbf{f}^{i n t}\left(\mathbf{u}_{g}\right)-\mathbf{f}^{e x t}\left(\mathbf{u}_{g}\right)=0
$$

where $\mathbf{u}_{g}$ is the global displacement vector. Since this equation is nonlinear in the displacements, an iterative procedure is required to obtain a solution. This is done using Newtons method. Before the exact solution is found, the internal and external forces are not in balance. Thus, we write

$$
\mathbf{f}^{i n t}\left(\mathbf{u}_{g}\right)-\mathbf{f}^{e x t}\left(\mathbf{u}_{g}\right)=\mathbf{r}\left(\mathbf{u}_{g}\right),
$$

where $\mathbf{r}$ is the imbalance force, the residual. Linearizing the residual and equating the result to zero gives

$$
\mathbf{r}\left(\mathbf{u}_{g}^{k}\right)+\frac{\partial \mathbf{r}\left(\mathbf{u}_{g}^{k}\right)}{\partial \mathbf{u}_{g}} \Delta \mathbf{u}_{g}=\mathbf{0}
$$


where $k$ refers to the iteration number. Solving for $\Delta \mathbf{u}_{g}$ gives the increment in displacement

$$
\Delta \mathbf{u}_{g}=-\left(\frac{\partial \mathbf{r}\left(\mathbf{u}_{g}^{k}\right)}{\partial \mathbf{u}_{g}}\right)^{-1} \mathbf{r}\left(\mathbf{u}_{g}^{k}\right) .
$$

The next estimate for the solution is then obtained from $\mathbf{u}^{k+1}=\mathbf{u}_{g}^{k}+\Delta \mathbf{u}_{g}$. This procedure is repeated until the solution is found at a prescribed accuracy. In the linearization process (21) we need to relate differentials of the internal and external forces to differentials of the nodal displacements. Using the superposed dot notation for convenience, this is written as

$$
\dot{\mathbf{f}}^{\text {int }}=\mathbf{K}_{g}^{\text {int }} \dot{\mathbf{u}}_{g} \quad \text { and } \quad \dot{\mathbf{f}}^{e x t}=\mathbf{K}_{g}^{e x t} \dot{\mathbf{u}}_{g} .
$$

The matrices $\mathbf{K}_{g}^{\text {int }}$ and $\mathbf{K}_{g}^{\text {ext }}$ will be formed next.

\subsection{Linearization of the internal forces}

We begin by linearizing the internal forces. The element internal nodal forces corresponding to node $I$ are computed according to

$$
\mathbf{f}_{I}^{\text {int }}=\int_{\Omega_{0}} \mathbf{B}_{I}^{T}\{\mathbf{S}\} \mathrm{d} \Omega_{0} .
$$

Differentiating this gives

$$
\dot{\mathbf{f}}_{I}^{i n t}=\int_{\Omega_{0}} \dot{\mathbf{B}}_{I}^{T}\{\mathbf{S}\} \mathrm{d} \Omega_{0}+\int_{\Omega_{0}} \mathbf{B}_{I}^{T}\{\dot{\mathbf{S}}\} \mathrm{d} \Omega_{0} .
$$

Since the integral is always taken in the undeformed reference configuration, the volume $\Omega_{0}$ remains constant. The derivative of $\mathbf{B}^{I}$ is found by differentiating Eq.(19). Then using the interpolated rate of deformation gradient (Eq. (18)) and the nodal velocities, and after some algebra taking the vector of nodal velocities outside the integral, allows the first terms on the right hand side of Eq. (26), combined from the contributions of one element $(I=1, \ldots, n)$, to be written as

$$
\int_{\Omega_{0}} \dot{\mathbf{B}}^{T}\{\mathbf{S}\} \mathrm{d} \Omega_{0}=\mathbf{K}^{g e o} \dot{\mathbf{u}}
$$

where the geometric stiffness matrix $\mathbf{K}^{\text {geo }}$ is

$$
\mathbf{K}^{g e o}=\int_{\Omega_{0}}\left[\begin{array}{ccc}
\mathbf{K}^{11} & \ldots & \mathbf{K}^{1 n} \\
\vdots & \ddots & \\
\mathbf{K}^{n 1} & \ldots & \mathbf{K}^{n n}
\end{array}\right] \mathrm{d} \Omega_{0}
$$

with

$$
\mathbf{K}^{I J}=\left[\begin{array}{cc}
K_{11}^{I J} & 0 \\
0 & K_{22}^{I J}
\end{array}\right] .
$$

Here, the diagonal elements of the submatrices $\mathbf{K}^{I J}$ are

$$
\begin{aligned}
K_{11}^{I J}= & N,{ }_{R}^{I} N,{ }_{R}^{J} S_{11}+N,{ }_{Z}^{I} N,{ }_{Z}^{J} S_{22}+ \\
& N^{I} N^{J} / R^{2} S_{33}+\left(N,{ }_{R}^{I} N,{ }_{Z}^{J}+N,{ }_{Z}^{I} N,{ }_{R}^{J}\right) S_{12}, \\
K_{22}^{I J}= & N,{ }_{R}^{I} N,{ }_{R}^{J} S_{11}+N,{ }_{Z}^{I} N,{ }_{Z}^{J} S_{22}+ \\
& \left(N,{ }_{R}^{I} N,{ }_{Z}^{J}+N,{ }_{Z}^{I} N,{ }_{R}^{J}\right) S_{12} .
\end{aligned}
$$


The second term on the right hand side of Eq. (26) can be formed by using the constitutive equation. Differentiation of the hyperelastic constitutive equation, Eq. (9), gives for the time derivative of $\mathbf{S}$ the expression

$$
\dot{\mathbf{S}}=\frac{\partial^{2} \psi}{\partial \mathbf{E} \partial \mathbf{E}}: \dot{\mathbf{E}}=\mathbf{C}^{S E}: \dot{\mathbf{E}} .
$$

Writing this in matrix form using Voigt notation and utilizing the expression $\{\dot{\mathbf{E}}\}=\mathbf{B} \dot{\mathbf{u}}$ for the rate of Green strain gives for the material stiffness matrix

$$
\mathbf{K}^{m a t}=\int_{\Omega_{0}} \mathbf{B}^{T}\left[\mathbf{C}^{S E}\right] \mathbf{B} \mathrm{d} \Omega_{0} .
$$

The total tangent stiffness is given by the sum of the geometric and material stiffnesses $\mathbf{K}^{i n t}=\mathbf{K}^{g e o}+\mathbf{K}^{\text {mat }}$. The global matrix $\mathbf{K}_{g}^{i n t}$ is assembled by joining the contributions from all elements.

\subsection{Linearization of the external forces}

The centrifugal force caused by the rotation of the roll is taken into account as a body force. The element nodal force for node $I$ due to a general body force $\mathbf{b}$ is

$$
\mathbf{f}_{I}^{e x t}=\int_{\Omega_{0}} N^{I} \rho_{0} \mathbf{b} \mathrm{d} \Omega_{0},
$$

where $\rho_{0}$ is the density of the material in the reference state. A constant angular velocity $\omega$ introduces the force $\mathbf{b}=\omega^{2} r \mathbf{e}_{r}$ per unit mass, where $r$ is the current radius. Since the body force depends on the current radius $r$, and is thus a function of the displacement, it has to be linearized for the equilibrium iteration process. By differentiating the expression for the external forces the load stiffness $\mathbf{K}^{\text {ext }}$ for one element due to the centrifugal force is found to be

$$
\mathbf{K}^{e x t}=\int_{\Omega_{0}} \mathbf{N}^{T}\left[\begin{array}{cc}
\omega^{2} & 0 \\
0 & 0
\end{array}\right] \mathbf{N} \mathrm{d} \Omega_{0},
$$

where

$$
\mathbf{N}=\left[\begin{array}{ccccc}
N^{1} & 0 & \ldots & N^{n} & 0 \\
0 & N^{1} & \ldots & 0 & N^{n}
\end{array}\right]
$$

The global matrix $\mathbf{K}_{g}^{e x t}$ is assembled by joining the contributions from all elements. However, since the cumulative process of building the roll requires a large number of steps in which elements are added to the roll, the displacements during one load step are small. Considering this, and the magnitudes of the roll angular velocities found in practice, the linearization of the centrifugal force is not mandatory. In many cases it is, in fact, computationally more efficient to discard the linearization of the centrifugal force from the Jacobian of the residual.

\section{Constitutive equations}

In many winding applications the displacements can be large and displacement gradients are not small compared to unity. In paper winding, for example, the radial strains of the rolls take typically values of the order $5-10 \%$, and even $15 \%$ in the case of certain soft paper boards. Therefore, the infinitesimal strain theory, utilized usually for strains well below $1 \%$, is not a good approximation for such rolls. In the present work finite strains are incorporated into the model by employing the total Lagrangian formulation. 
Most of the thin sheet media wound into rolls exhibit orthotropic material symmetry. The symmetry group for an orthotropic material is generated by the set of reflections $\mathbf{R}^{(a)}$, $\mathbf{R}^{(b)}$, and $\mathbf{R}^{(c)}$ in three orthogonal planes normal to the mutually orthogonal vectors $\mathbf{a}$, $\mathbf{b}$, and $\mathbf{c}$ specifying the directions of orthotropy of the material. The symmetry group is therefore comprised of the tensors

$$
\begin{aligned}
& \mathbf{I}, \mathbf{R}^{(a)}, \mathbf{R}^{(b)}, \mathbf{R}^{(c)}, \mathbf{Q}^{(a)}(\pi)=\mathbf{R}^{(b)} \cdot \mathbf{R}^{(c)} \\
& \mathbf{Q}^{(b)}(\pi)=\mathbf{R}^{(c)} \cdot \mathbf{R}^{(a)}, \mathbf{Q}^{(c)}(\pi)=\mathbf{R}^{(a)} \cdot \mathbf{R}^{(b)}, \\
& -\mathbf{I}=\mathbf{R}^{(a)} \cdot \mathbf{R}^{(b)} \cdot \mathbf{R}^{(c)}
\end{aligned}
$$

where $\mathbf{Q}^{(a)}(\pi), \mathbf{Q}^{(b)}(\pi)$ and $\mathbf{Q}^{(c)}(\pi)$ denote rotations through an angle $\pi$ about the directions of $\mathbf{a}, \mathbf{b}$, and $\mathbf{c}$, respectively. In the present work the wound roll is modeled as a hyperelastic material so that an elastic potential function exists. The stress-strain relation may then be written as

$$
S_{I J}=\frac{\partial \psi(\mathbf{E})}{\partial E_{I J}}
$$

where $S_{I J}$ and $E_{I J}$ are the components of the second Piola-Kirchhoff stress tensor and the Green strain tensor, respectively, and $\psi$ the strain energy per unit undeformed volume (symmetrized in $E_{I J}$ and $E_{J I}$ ). For an orthotropic hyperelastic material, $\psi(\mathbf{E})$ must be invariant under each of the transformations (37). It is well known from group theoretical considerations that any scalar invariant may be expressed as a single-valued function of the elements of the irreducible integrity basis. The irreducible integrity basis, on the other hand, consists of a finite number of polynomial scalar invariants of the tensor, none of which is expressible as a polynomial in the remaining ones. It has been shown in [20] that the irreducible integrity basis for the tensor $\mathbf{E}$ in the case of orthotropic material symmetry consists of the seven invariants

$$
\begin{aligned}
& \left.I_{1}=\operatorname{tr} \mathbf{E}, \quad I_{2}=\frac{1}{2}\left((\operatorname{tr} \mathbf{E})^{2}-\operatorname{tr} \mathbf{E}^{2}\right)\right), \quad I_{3}=\operatorname{det} \mathbf{E} \\
& I_{4}=\mathbf{a} \cdot \mathbf{E} \cdot \mathbf{a}, \quad I_{5}=\mathbf{a} \cdot \mathbf{E}^{2} \cdot \mathbf{a} \\
& I_{6}=\mathbf{b} \cdot \mathbf{E} \cdot \mathbf{b}, \quad I_{7}=\mathbf{b} \cdot \mathbf{E}^{2} \cdot \mathbf{b}
\end{aligned}
$$

Note that the directions of the vectors $\mathbf{a}$ and $\mathbf{b}$, which characterize the anisotropy of the material, may vary from point to point corresponding to the case where the fibres are arranged along curves which are not straight lines. For wound rolls the material directions are $\mathbf{a}=\mathbf{e}_{R}$ and $\mathbf{b}=\mathbf{e}_{\Theta}$, where $\mathbf{e}_{R}$ and $\mathbf{e}_{\Theta}$, unit vectors of the cylindrical coordinate system, are normal to two planes of reflectional symmetry of the roll in the reference (or undeformed) configuration. For the orthotropic hyperelastic material, then, the strain-energy $\psi=\psi(\mathbf{E})$ can depend on $\mathbf{E}$ only through these seven invariants so that

$$
\psi=\psi\left(I_{1}, I_{2}, \ldots, I_{7}\right)
$$

A strain-energy function of this form ensures the material frame indifference (objectivity) and orthotropic material symmetry properties of the constitutive equations (38). In this work, the deformation of the roll is restricted to axisymmetric cases. A straightforward calculation shows that for axisymmetric cases $\left(E_{R \Theta}=E_{\Theta Z}=0\right)$ the invariants (39) become

$$
\begin{aligned}
I_{1} & =E_{R R}+E_{Z Z}+E_{\Theta \Theta}, \\
I_{2} & =E_{R R} E_{Z Z}+E_{R R} E_{\Theta \Theta}+E_{Z Z} E_{\Theta \Theta}-E_{R Z}^{2}, \\
I_{3} & =E_{R R} E_{Z Z} E_{\Theta \Theta}-E_{R Z}^{2} E_{\Theta \Theta},
\end{aligned}
$$




$$
\begin{aligned}
I_{4} & =E_{R R}, \\
I_{5} & =E_{R R}^{2}+E_{R Z}^{2}, \\
I_{6} & =E_{\Theta \Theta}, \\
I_{7} & =E_{\Theta \Theta}^{2} .
\end{aligned}
$$

It can be easily seen from equations (41) that, in this axisymmetric case, the invariants $I_{2}$, $I_{3}$ and $I_{7}$ can be expressed as polynomials in the invariants $I_{1}, I_{4}, I_{5}$ and $I_{6}$. The remaining relations can be written in the form

$$
\begin{aligned}
& I_{4}=E_{R R}, \quad I_{6}=E_{\Theta \Theta}, \\
& I_{1}-I_{4}-I_{6}=E_{Z Z}, \quad I_{5}-I_{4}^{2}=E_{R Z}^{2} .
\end{aligned}
$$

The most straightforward way to create the constitutive equations would be the specification of the functional dependence of the strain energy on the invariants $I_{1}, I_{4}, I_{5}$ and $I_{6}$. In the following, however, a slightly different approach is chosen. The motivation for this approach is that in the small strain limit it restores a well-established constitutive relation used by several authors. The starting point is the complementary strain energy $\psi^{*}=\psi^{*}(\mathbf{S})$ in the form

$$
\begin{aligned}
\psi^{*}(\mathbf{S}) & =\frac{C_{0}}{C_{1}^{2}}\left(1-\frac{C_{1}}{C_{0}} S_{R R}\right)\left[\ln \left(1-\frac{C_{1}}{C_{0}} S_{R R}\right)-1\right] \\
& +A_{R \Theta} S_{R R} S_{\Theta \Theta}+A_{R Z} S_{R R} S_{Z Z}+A_{\Theta Z} S_{\Theta \Theta} S_{Z Z} \\
& +\frac{1}{2} A_{\Theta \Theta} S_{\Theta \Theta}^{2}+\frac{1}{2} A_{Z Z} S_{Z Z}^{2}+\frac{1}{8} B_{R Z}\left(S_{R Z}+S_{Z R}\right)^{2}
\end{aligned}
$$

The corresponding strain-stress relation $E_{I J}=\partial \psi^{*} / \partial S_{I J}$ becomes

$$
\begin{aligned}
E_{R R} & =-\frac{1}{C_{1}} \ln \left(1-\frac{C_{1}}{C_{0}} S_{R R}\right)+A_{R \Theta} S_{\Theta \Theta}+A_{R Z} S_{Z Z}, \\
E_{\Theta \Theta} & =A_{R \Theta} S_{R R}+A_{\Theta \Theta} S_{\Theta \Theta}+A_{\Theta Z} S_{Z Z}, \\
E_{Z Z} & =A_{R Z} S_{R R}+A_{\Theta Z} S_{\Theta \Theta}+A_{Z Z} S_{Z Z}, \\
E_{R Z} & =\frac{1}{2} B_{R Z} S_{R Z} .
\end{aligned}
$$

The first three equations can be (numerically) inverted to give the relations

$S_{R R}=S_{R R}\left(E_{R R}, E_{\Theta \Theta}, E_{Z Z}\right), S_{\Theta \Theta}=S_{\Theta \Theta}\left(E_{R R}, E_{\Theta \Theta}, E_{Z Z}\right)$ and $S_{Z Z}=S_{Z Z}\left(E_{R R}, E_{\Theta \Theta}, E_{Z Z}\right)$. The fourth equation determines the relation $S_{R Z}=2 B_{R Z}^{-1} E_{R Z}$. Noting that $\mathbf{S}$ and $\mathbf{E}$ are conjugate variables and by utilizing the proper Legendre transformation, the corresponding strain energy $\psi=\psi(\mathbf{E})$ is obtained as

$$
\psi(\mathbf{E})=S_{R R} E_{R R}+S_{\Theta \Theta} E_{\Theta \Theta}+2 S_{R Z} E_{R Z}-\psi^{*}(\mathbf{S}(\mathbf{E})) .
$$

It can now be easily seen by the aid of equations (42) and (44) that the strain-energy (45) is of the form

$$
\begin{aligned}
\psi & =\psi\left(E_{R R}, E_{\Theta \Theta}, E_{Z Z}, E_{R Z}^{2}\right) \\
& =\psi\left(I_{4}, I_{6}, I_{1}-I_{4}-I_{6}, I_{5}-I_{4}^{2}\right) \equiv \tilde{\psi}\left(I_{1}, I_{4}, I_{5}, I_{6}\right) .
\end{aligned}
$$

Thus, the constitutive equations (44) can be derived from a strain-energy function (46), which is a single-valued function of the elements of the irreducible integrity basis. Consequently, the material frame indifference and orthotropic symmetry requirements for a finite 
strain analysis are fulfilled. Finally, it will be noted that the incremental form of equations $(44)$ is

$$
\begin{aligned}
\Delta E_{R R} & =\frac{1}{C_{0}-C_{1} S_{R R}} \Delta S_{R R}+A_{R \Theta} \Delta S_{\Theta \Theta}+A_{R Z} \Delta S_{Z Z} \\
\Delta E_{\Theta \Theta} & =A_{R \Theta} \Delta S_{R R}+A_{\Theta \Theta} \Delta S_{\Theta \Theta}+A_{\Theta Z} \Delta S_{Z Z} \\
\Delta E_{Z Z} & =A_{R Z} \Delta S_{R R}+A_{\Theta Z} \Delta S_{\Theta \Theta}+A_{Z Z} \Delta S_{Z Z} \\
\Delta E_{R Z} & =\frac{1}{2} B_{R Z} \Delta S_{R Z} .
\end{aligned}
$$

A similar form or its counterpart for one-dimensional models has been used by several authors in small strain wound roll models $[4,10]$. The first term on the right hand side of the first equation corresponds to the tangent radial modulus $E_{r}=C_{0}-C_{1} \sigma_{r}$, which was introduced by Pfeiffer in a one-dimensional small strain winding analysis [21]. In the small strain regime, the elastic constants of equation (47) correspond to the conventional engineering constants as shown in Table 1 . Here $E_{\theta}$ and $E_{z}$ are the elastic moduli of the

Table 1: Correspondence between the current material parameters and the engineering elastic constants.

\begin{tabular}{cccc}
\hline Current & Engineering & Current & Engineering \\
\hline$A_{\Theta \Theta}$ & $1 / E_{\theta}$ & $A_{R \Theta}$ & $-\nu_{\theta r} / E_{\theta}$ \\
$A_{Z Z}$ & $1 / E_{z}$ & $A_{R Z}$ & $-\nu_{z r} / E_{z}$ \\
$B_{R Z}$ & $1 / G_{r z}$ & $A_{\Theta Z}$ & $-\nu_{z \theta} / E_{z}$ \\
\hline
\end{tabular}

roll in the circumferential and axial directions, $G_{r z}$ is the shear modulus in the $r z$-plane, and $\nu_{\theta r}, \nu_{z r}, \nu_{z \theta}$ are the Poisson's ratios of the roll.

\section{Reference configuration}

The characterisation of deformation requires an initial or reference configuration, to which the current configuration is compared. In the present case, the most convenient choice is to consider the state of the incoming web before it is attached to the roll as the reference state. The refence state and the steps in the process of adding a new layer to the roll are depicted in Fig. 2. Since each added layer has its own initial displacement and strain state at the time when it becomes part of the growing roll, the strain field of the roll is, in general, discontinuous. After the attachment of a new layer, the roll and the new layer form a continuous medium and the displacements from here on are continuous.

In the present model, the process of adding a new layer on the roll can be divided into three steps.

- First, the reference state of the new layer is a cylindrical shell with a circumferential strain equal to the strain of the incoming web and with an inner radius $r_{a}$ and shell thickness $h$. The dimension $r_{a}$ is the average radius of the current roll outer surface as shown in Fig 2.

- Second, displacements of the inner surface of the new layer are prescribed so that they bring this surface in contact with the current roll outer surface. The surfaces are fixed together so that from here on their displacements are equal. 


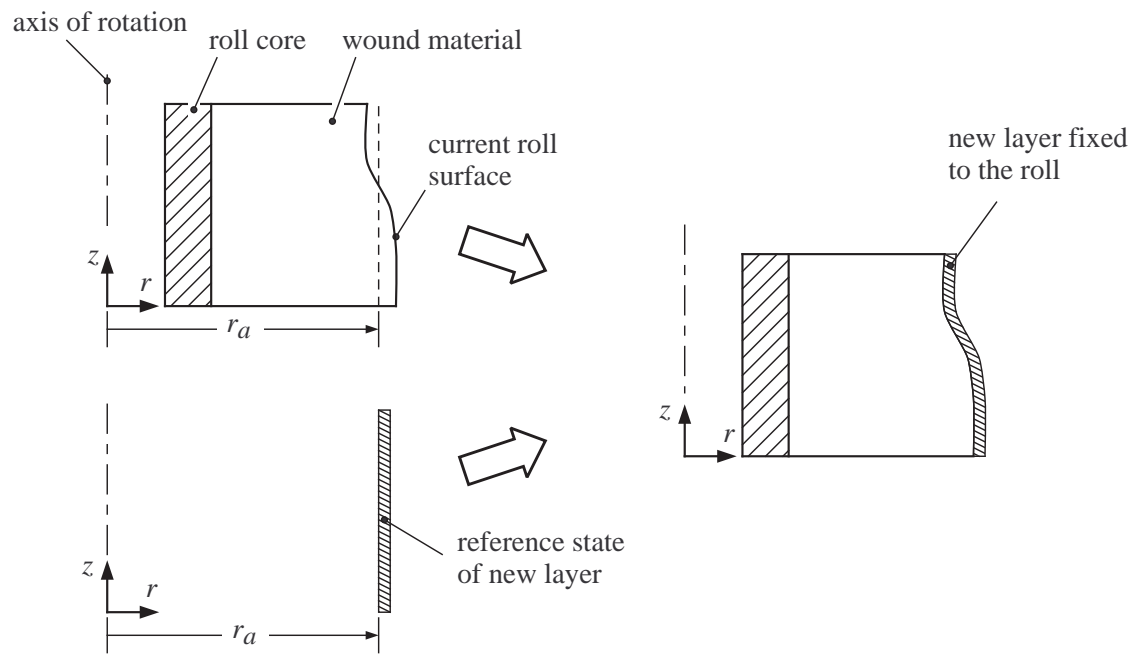

Figure 2: Process of adding a new layer to the roll. The average radius of the deformed roll surface is $r_{a}$

- Third, the new layer is allowed to tighten around the current roll and the corresponding FE-equations are solved to find the new equilibrium state of stress and deformation for the new roll (including the new layer).

\section{$7 \quad$ Numerical examples}

In this section calculated examples are given. First, the present model is compared to a well-established 1D-model. Then, the effect of the FE-discretization is considered. Second, the effect of the centrifugal force due to the rotation of the wound roll during winding is demonstrated in a plane strain case. Third, examples of the effect of the finite axial length of the roll are presented. Cases where the tension of the incoming web is either constant or linearly varying in the web width direction are considered. The values for the material parameters used in the calculations are given in Appendix I. Values measured from catalog paper are used for the paper roll and values from [22] for the core material. The finite element discretization was done using four-noded bilinear elements. The stress measure used in the following examples is the Cauchy stress (true stress), which is obtained from the second Piola-Kirchhoff stress by the formula $\boldsymbol{\sigma}=J^{-1} \mathbf{F} \cdot \mathbf{S} \cdot \mathbf{F}^{T}$, where $J=\operatorname{det} \mathbf{F}$.

\subsection{Comparison to Hakiel model}

Results calculated using the present winding model are compared to results by the well known winding model proposed by Hakiel [10]. The Hakiel model is one-dimensional, the only space variable being the radial coordinate. For this reason an assumption of either plane stress or plane strain condition has to be made. To get comparable results with the current model, the axial width of the roll was given a small value of $0.2 \mathrm{~mm}$, which practically enforces a state of plane stress in the roll. Note that in the plane stress case, the value of the axial modulus $E_{z}$ has no effect on the results. In the test, a web with thickness of $60 \mu \mathrm{m}$ with an incoming tension of $600 \mathrm{~N} / \mathrm{m}$ is wound on the roll core. One finite element per paper layer was used. In Fig. 3 the calculated stresses from a 2811-lap 
(a)

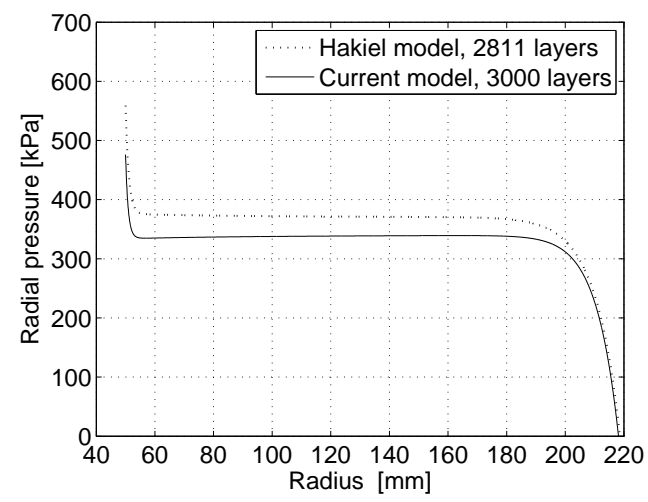

(b)

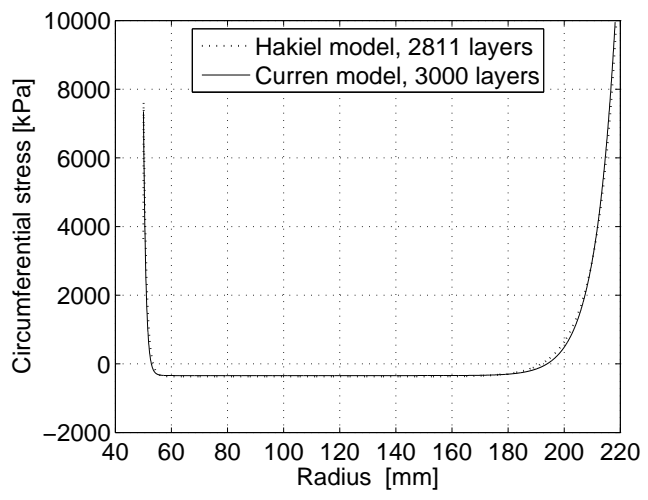

Figure 3: Radial pressure $p$ (a) and circumferential stress $\sigma_{\theta}$ (b) in the wound roll calculated using the Hakiel and present models.

simulation of the Hakiel model are compared to a 3000-lap simulation with the present model. The compression of the existing roll as a new layer is added on is not accounted for by the Hakiel model. After winding 2811 layers, an outer radius of $r=218.5 \mathrm{~mm}$ is predicted by the Hakiel model. When using the current model, where the compression of the layers is accounted for, it takes 3000 laps to get the same outer radius for the roll. This corresponds to an increase of almost $7 \%$ in the revolutions of web material in the roll. It can be seen from Fig. 3 that the circumferential stresses of the two models almost coincide. There is a more pronounced difference between the radial pressures. The values given by the Hakiel model are clearly higher. The difference is due to the accumulated effect of the tension differences of each separate lap and the pressure dependence of the radial modulus of elasticity. It must be noted that the fundamental difference between the plane stress (or 1D) version of the present model and the Hakiel model lies in the treatment of the roll deformation during winding. In the Hakiel model, the radial locations of the layers in the balance equations are kept constant, whereas in the present model they are continuously updated due to the use of large deformation theory in the roll model. In fact, the plane stress version of the present model is very close to the Benson model [12], which also updates the layer locations in the roll every time a new lap is added. This is also supported by the comparison of the Benson and Hakiel models, which shows that the results by the Benson model deviate from those by the Hakiel model in a very similar manner as seen here.

The calculations indicated that the compressive radial strain in the roll calculated using the present model increases from zero at the roll surface to over $8 \%$ at the roll core. This suggests that large strain continuum mechanics should be used when modelling paper rolls since the validity of the small strain theory is typically limited to strain values well below $1 \%$.

\subsection{Effect of mesh density}

Since the finite element model becomes computationally heavier after the addition of each layer, the computation time depends primarily on the number of layers wound onto the roll. Significant savings in computation time can be gained if, instead of adding one element layer for each paper layer, a model element layer spanning several physical paper layers is added. To find a suitable balance between the computation time and accuracy, the calculation was repeated using one finite element layer for one, two, four and six paper 
layers. The results are shown in Fig. 4. The top line shows the reference solution, calculated using one element layer for each paper layer. As the number of paper layers for each element layer increases, the solution drifts further away from the reference solution. As can be seen from the figure, the radial pressure is much more sensitive to the number of paper layers in one element layer than the circumferential stress. In particular, the maximum pressure at the core takes the values $475,465,440$ and $420 \mathrm{kPa}$ correponding to a $0,2.1,7.3$ and $11.6 \%$ relative decrease in the maximum pressure, respectively.

(a)

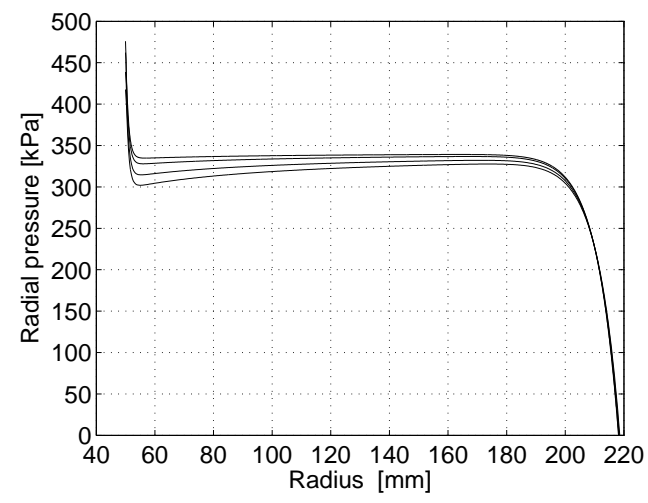

(b)

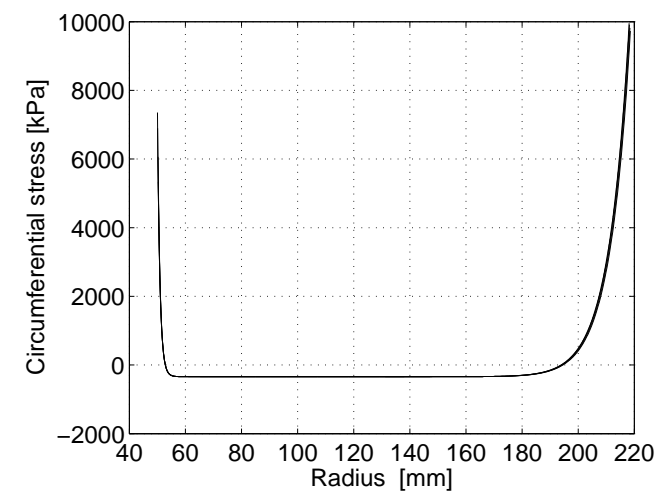

Figure 4: Radial pressure $p$ (a) and circumferential stress $\sigma_{\theta}$ (b) in the wound roll with one finite element layer representing one, two, four and six paper layers (from top to bottom, respectively).

\subsection{Effect of centrifugal forces}

Modern high speed winders can be run at web speeds of $50 \mathrm{~m} / \mathrm{s}$. With a radius of $300 \mathrm{~mm}$, for example, this corresponds to an angular velocity of almost $170 \mathrm{rad} / \mathrm{s}$. Since the centrifugal body force acting on the rotating paper roll is proportional to the square of the angular velocity, high forces are bound to be generated. The centrifugal force during winding tends to decrease the compressive radial pressure and tensional circumferential stress in the finished nonrotating roll due to the radial shrinking of the roll when the roll rotation is stopped and the centrifugal force ceases to affect. For a trouble-free unwinding, it would be beneficial if the pressure and web tension distributions in the roll were as constant as possible in the radial direction. Since the centrifugal force tends to decrease the radial pressure in the finished roll, it is evident that increasing the wound-in tension could at least partially compensate for the loosening effect of the centrifugal force. A first approximation for the required compensative winding force can be developed by noting that the radial pressure under the topmost layer of the paper roll is

$$
p=\frac{T_{\text {in }}}{R}-\rho \omega^{2} h R
$$

where $T_{i n}$ is the incoming web tension, $R$ the current radius of the roll, $\rho$ the paper density and $h$ the web thickness. In the case $\omega=0$ and $T_{i n}=T_{0}$ this reduces to $p=T_{0} / R$. It can be seen that by using a compensated incoming web tension

$$
T_{i n}=T_{0}+\rho h \omega^{2} R^{2}
$$


(a)

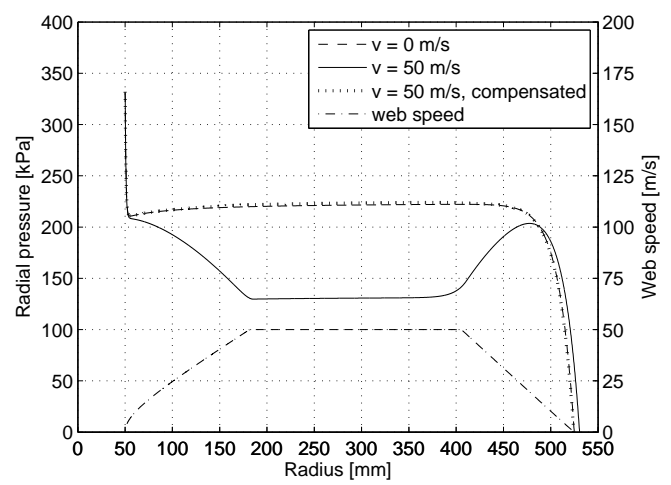

(b)

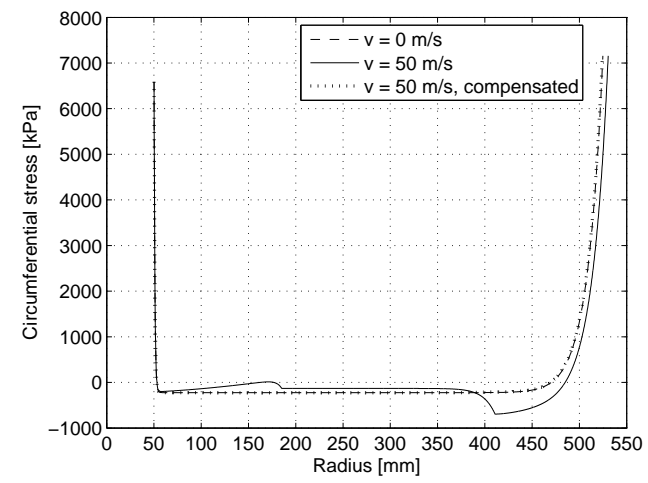

Figure 5: Radial pressure $p$ and the speed of the incoming web as a function of the roll radius (a) and circumferential stress $\sigma_{\theta}$ (b) in the finished nonrotating roll for three different cases (see the text).

the radial pressure under the topmost layer of the rotating case equals that of the nonrotating and noncompensated case. To see how well the compensation works, a test case was calculated. In the test case the web speed during winding was first accelerated from zero to $50 \mathrm{~m} / \mathrm{s}$ using an acceleration $1.0 \mathrm{~m} / \mathrm{s}^{2}$. After this the web speed remained constant at $50 \mathrm{~m} / \mathrm{s}$. Finally, the roll angular velocity was restored back to zero during the last 1500 roll revolutions by decreasing the angular velocity linearly as a function of roll rotations. The web speed as a function of the roll radius is shown in Fig. 5 (a). The finished roll had 6000 paper layers and an outer diameter of approximately $1050 \mathrm{~mm}$. One finite element per paper layer was used in the calculation.

The radial pressure distributions for three different winding conditions are shown in Fig. 5 (a). The dashed line is calculated using a constant incoming web tension of $600 \mathrm{~N} / \mathrm{m}$ and zero (or very low) winding speed. The typical plateau in the radial pressure, familiar already from the Hakiel model [10], can be seen. The solid line is calculated using the same constant incoming web tension $600 \mathrm{~N} / \mathrm{m}$ and the winding velocity profile described above. The decrease of the plateau value due to the centrifugal force is clearly visible. Finally, the dotted line is calculated using the compensated incoming web tension proposed by Eq. (49). It can be seen from the figure that adding the compensation term effectively evens out the effect of the centrifugal force, and a final radial pressure distribution almost equal to that of the nonrotating (or slowly rotating) case is obtained.

The circumferential stresses in the roll for the aforementioned calculation cases are shown in Fig. 5 (b). Again the stress distributions in the zero winding speed (dashed line) and the tension compensated (dotted line) cases are practically identical. The effect of the centrifugal force on the circumferential stress is clearly displayed by the solid line. It can be seen from Fig. 5 that the outer radius of the finished roll is larger in the case of the solid line. This is as expected since, due to the centrifugal force, the pressure in the finished roll becomes smaller and, therefore, the paper layers are less compressed in the radial direction.

\subsection{Uniform incoming web tension}

Comparison of the roll stresses for zero and nonzero Poisson's ratios of the web and the effect of the axial dimension of the roll on the stresses are considered next. In the 
calculations the width of the web was $300 \mathrm{~mm}$, the roll consisted of 3000 paper layers of initial (non-stressed) thickness $83 \mu \mathrm{m}$, and 600 model layers (FE-layers) were used. The calculations indicated that the results were not especially sensitive to the element length in the axial direction, except near the roll ends, where the mesh has to be fine enough to capture the edge effects. A total of 34 elements in the roll axial direction were used (see Fig. 6). Due to the symmetry of the system and the external load, the radial, circumferential and axial stresses are symmetric with respect to the roll's central cross section, while the shear stress is antisymmetric.

First, a simple case with a constant incoming web tension of $600 \mathrm{~N} / \mathrm{m}$ in the web width direction and with the values of the Poisson's ratios of the web set to zero was studied. Plots of the radial and circumferential stress distributions for the finished roll are shown in Fig. 6. The resuts are practically identical to those of the corresponding one-dimensional plane stress model, and the calculations showed that the stress distributions along radial lines are practically independent of the position of the line at the $z$-axis. The axial and shear stresses are, in concordance with one-dimensional plane stress models, practically zero in the whole roll except very near the roll core. Within the 100 undermost layers the axial stress changes from zero at the hundredth layer to approximately $530 \mathrm{kPa}$ at the core along the whole roll axis. The shear stress at the core changes from almost zero at $z=40 \mathrm{~mm}$ to $50 \mathrm{kPa}$ at the roll end (or $-50 \mathrm{kPa}$ at the other end). These stresses arise due to the elongation of the roll core in the axial direction under the stress of the undermost paper layer, which leads to small axial displacements in the roll near the core. A similar result is obtained for a magnetic tape in [5], where the axial and shear stresses are significant only near the web-core interface, and rapidly fall to almost zero elsewhere.

(a)

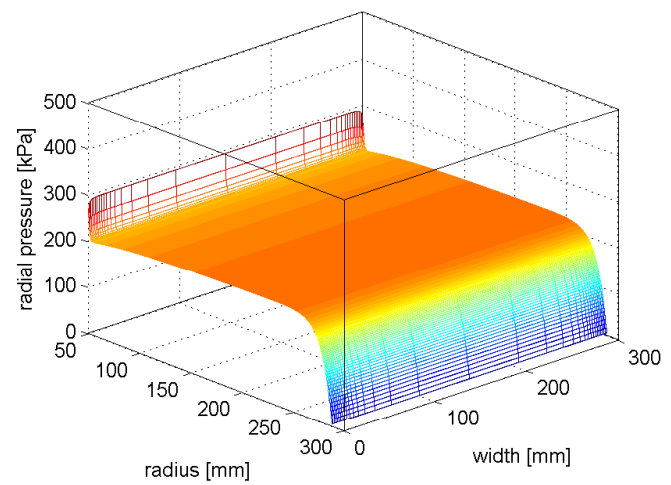

(b)

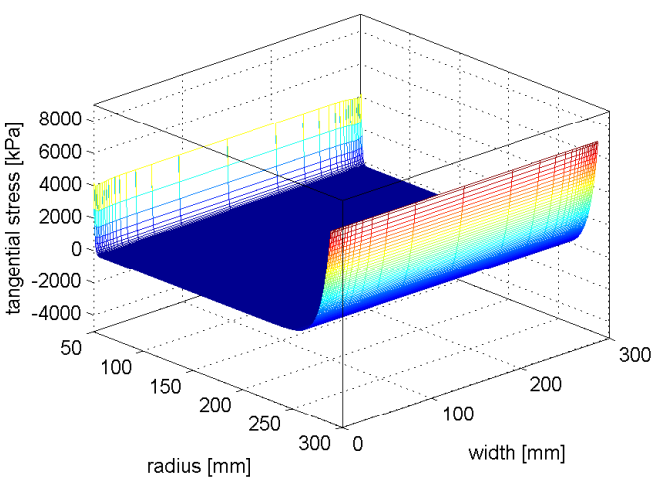

Figure 6: Radial pressure $p$ (a) and circumferential stress $\sigma_{\theta}$ (b) in the wound roll when the incoming web tension is constant across the web width and the Poisson's ratios are set to zero.

Second, the Poisson's ratios of the web were set to the nonzero values $\nu_{\theta z}=0.3, \nu_{\theta r}=$ $1.71, \nu_{z r}=0.39$, while the values of the other parameters were kept the same. Plots of the stress distributions for the finished roll are presented in Figs. 7 and 8. It can be seen from Figs. 6 (a) and 7 (a) that the radial pressures in the two cases behave qualitatively in a similar manner although the pressures in the latter case are slightly higher. The difference is about $5 \%$ within the plateau area. A closer examination of Fig. 7 (a) also shows a small increase (about $5 \%$ ) in the radial pressure of the plateau area within a $40 \mathrm{~mm}$ zone near 
(a)

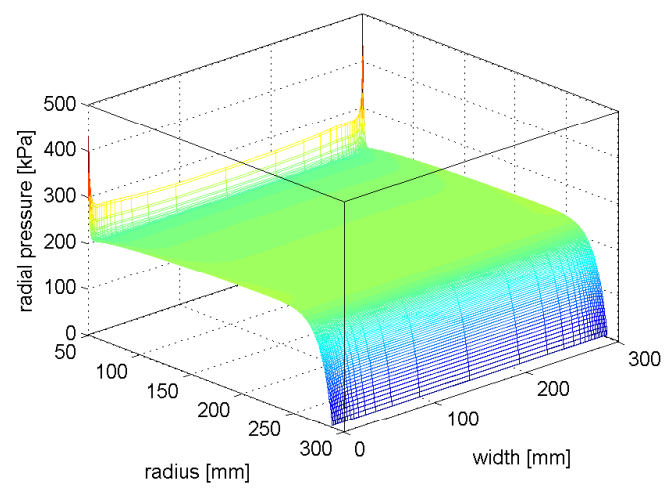

(b)

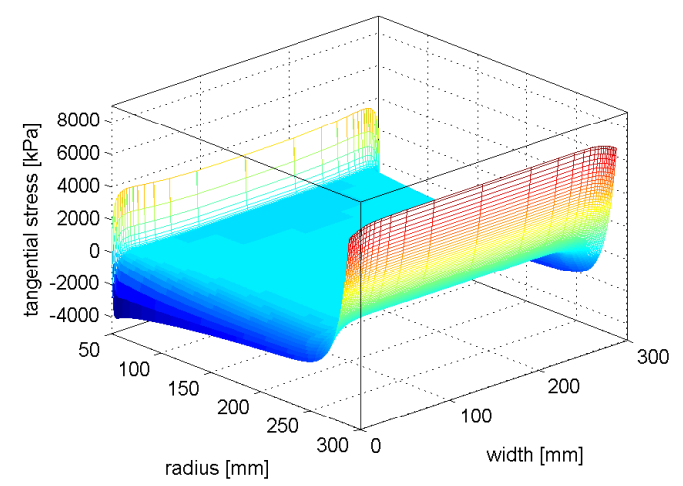

Figure 7: Radial pressure $p$ (a) and circumferential stress $\sigma_{\theta}(\mathrm{b})$ in the wound roll when the incoming web tension is constant across the web width and the Poisson's ratios are nonzero.

(a)

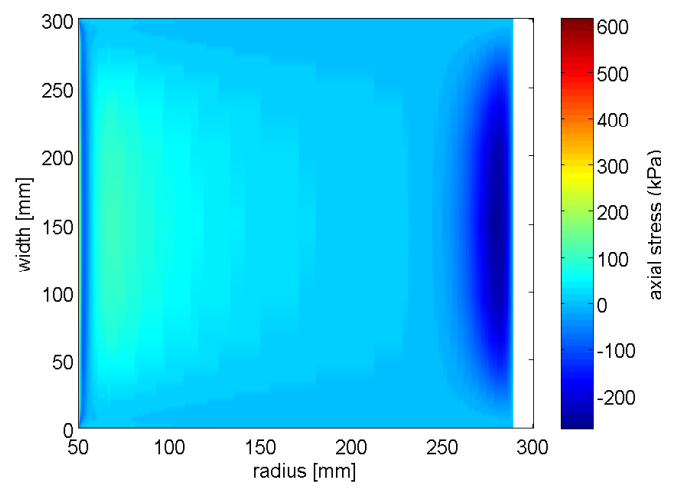

(b)

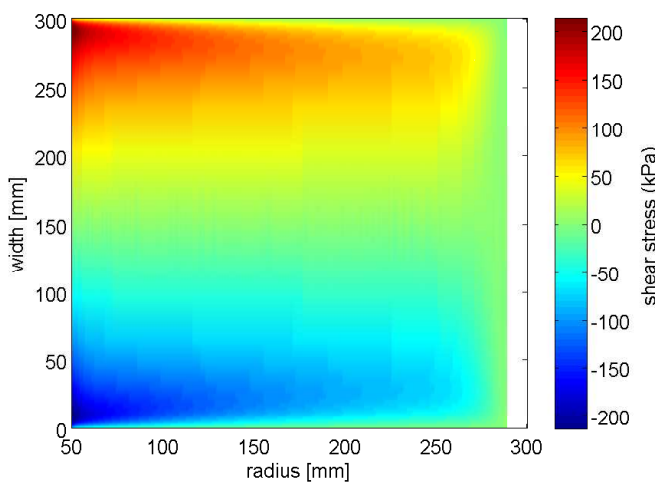

Figure 8: Axial stress $\sigma_{z}$ (a) and shear stress $\tau_{r z}$ (b) in the wound roll when the incoming web tension is constant across the web width and the Poisson's ratios are nonzero.

the roll ends. This edge effect is much more pronounced in the case of Fig 7 (a) than of Fig. 6 (a). A corresponding edge effect has been found in [5], where the cross-width variation of the radial pressure in a magnetic tape is about $10 \%$ near the roll core. In Fig. 7 (a) anomalously high pressure values (the red spikes) are found at the core near the roll ends. It is well known that at a point where three dissimilar materials join (here paper, roll core and ambient air), a stress singularity will appear [23, 24]. The same phenomenon was also noticed by Lee and Wickert [5] in their two-dimensional roll model, and a free-edge corner stress singularity is also known to exist in composite laminates [25]. In a real roll, the stresses are limited by the yield strength of the materials, and in the case of a paper roll, in particular, by the slippage between the paper layers. When moving further away from the singular points the stress levels are rapidly smoothened and usable (converged) results are obtained. Comparison of Fig. 6 (b) with Fig. 7 (b) shows a strong edge effect in the latter. Compressive circumferential stresses exceeding $3000 \mathrm{kPa}$ are found at the roll ends. These stresses strongly increase the liability of the paper layers to buckling at the roll ends. This can also lead to a so called spoking defect in which the layers buckle to such an extent that 
an internal gap will be formed. It is well known that such a buckling is facilitated by the combined conditions of a low radial pressure and high compressive circumferential stress [5]. In Fig. 8 (a) a positive bump of height $100 \mathrm{kPa}$ and a negative bump of depth $250 \mathrm{kPa}$ in the axial stress can be seen near the core and roll surface, respectively. Similarly to the previous case of zero Poisson's ratios, the axial stress goes up to approximately $500 \mathrm{kPa}$, mainly due to the strain mismatch of the roll bottom and core surface, within the last 100 layers at the core. A closer examination reveals also small edge effects in the axial stress. In Fig. 8 (b) high shear stresses and shear stress gradients, especially near the roll edges, can be seen. The nonzero axial and shear stresses originate from the non-constant values of the radial and circumferential stresses along the roll radii. Since the radial and circumferential stresses are different at different radii, the nonzero Poisson's ratios give rise to different axial strains at different radii. This, in turn, results in the development of axial and shear stresses. In addition, since the Poisson's ratios of the roll and core are different, a tendency for the development of different axial strains in the undermost layers of the roll and surface of the roll core appears. Since the roll core and the undermost paper layer are stuck together by friction, axial and shear stresses will be developed in the immediate vicinity of the core surface. It is known from paper mills that a roll defect, the so called core burst, can take place near the roll edges [2]. In a core burst, the layers near the roll bottom burst out from the roll end leading to a severe roll defect. The emergence of a core burst can be understood in terms of the high shear stresses near the roll ends (see Fig. 8 (b)). The maximum absolute value for the shear stress is set by the friction limit $\mu p$. When the shear stress exceeds this limit, a rearrangement of the stresses occurs. If the friction limit is exceeded only within a small area, the shear and axial stresses nearby will conform to the situation and the balance in the roll will be restored. If, however, the friction limit is exceeded within an area large enough, the restoring capacity of the roll stresses may be insufficient. As a result, the layers near the core rush out from the roll leading to a core burst. Finally, an explanation for the high compressive circumferential stresses near the roll edges (see Fig. 7 (b)) will be given. It can be readily seen from the radial equilibrium equation that when the radial stress is almost constant, as is the case in Fig. 7 (a), an increment of the circumferential stress in the axial direction becomes approximately

$$
\Delta \sigma_{\theta} \approx \Delta\left(\frac{\partial \tau_{r z}}{\partial z}\right)=\frac{\partial^{2} \tau_{r z}}{\partial z^{2}} \Delta z
$$

The second derivative of $\tau_{r z}$ with respect to $z$ turns out to be increasingly negative towards the roll end (see Fig. 8 (b)), which together with equation (50) explains the edge behaviour of the circumferential stress in Fig. 7 (b). It should be noted that this behaviour is different from what could be expected from one-dimensional roll models. In one-dimensional roll models the radial equilibrium allows an interplay between only the radial and circumferential stresses (usually lower radial pressure implies higher circumferential compression). In the present model, however, the radial stress may remain almost as constant, and still strong changes in the circumferential stress may occur due to changes in the shear stress. Here the value of a numerical roll model is emphasized, since only the radial stress can be easily measured from real rolls.

\subsection{Nonuniform web tension}

In practice, the tension of the incoming web is not necessarily constant throughout the width of the web. Misalignment of the rollers, variations in the material properties and moisture content of the web, and a varying web thickness profile, for example, can all contribute to the tension distribution of the incoming paper web. To study the effect of 
(a)

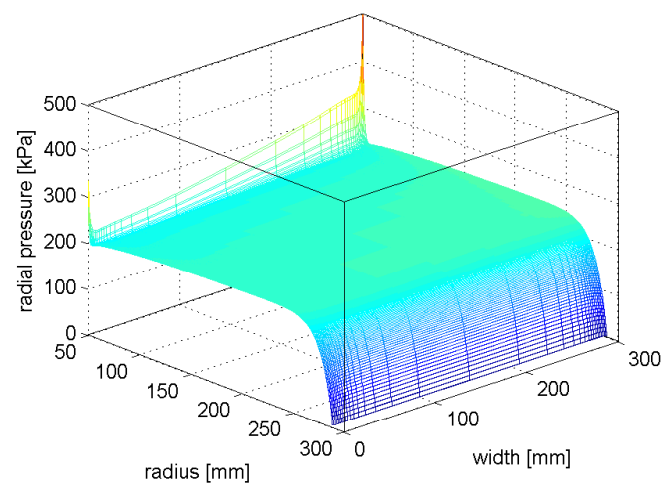

(b)

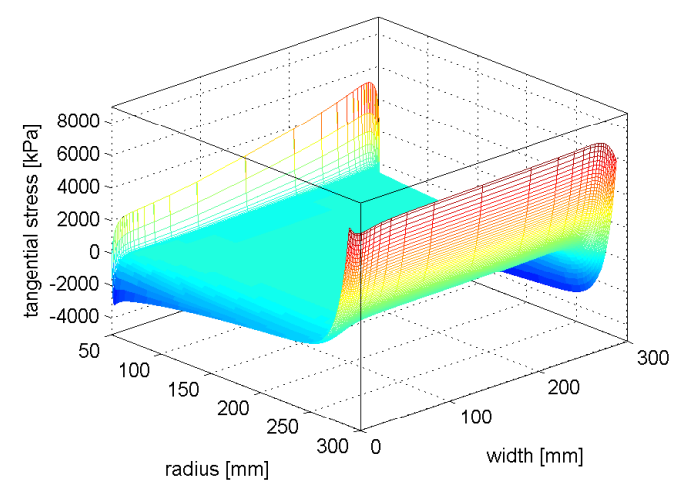

Figure 9: Radial pressure $p$ (a) and circumferential stress $\sigma_{\theta}$ (b) in the wound roll when the incoming web tension is linearly distributed across the web width.

(a)

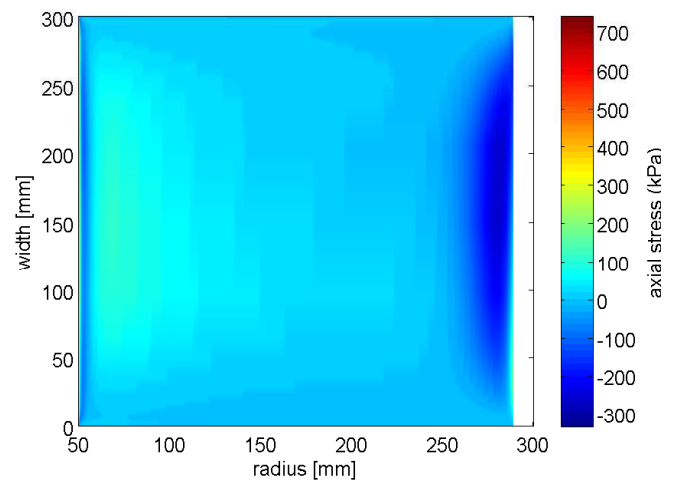

(b)

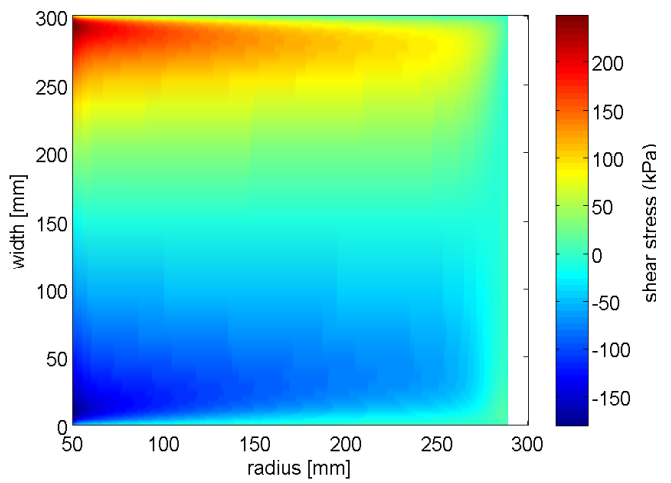

Figure 10: Axial stress $\sigma_{z}$ (a) and shear stress $\tau_{r z}$ (b) in the wound roll when the incoming web tension is linearly distributed across the web width.

a non-uniform incoming web tension on the stress distribution of the finished paper roll, a case with a linearly varying tension distribution across the web width was studied. The incoming web tension ranged from $400 \mathrm{~N} / \mathrm{m}$ at $z=0$ (left end) to $800 \mathrm{~N} / \mathrm{m}$ at $z=300 \mathrm{~mm}$ (right end) corresponding to the circumferential stresses 4.8 MPa and 9.6 MPa, respectively. The values of all other parameters were the same as in the previous calculation of uniform incoming web tension with nonzero Poisson's ratios. The radial and circumferential stress distributions for the finished roll are shown in Fig. 9. The effect of the linearly increasing incoming web tension is clearly visible in the vicinity of the roll core, where an almost linear distribution for the radial pressure and circumferential stress can be seen. As more layers are wound onto the roll, these stresses are, however, remarkably evened out in the roll axial direction. As can be seen from Fig. 9, already at a few millimeters (about 40 paper layers) away from the roll core the radial and circumferential stresses are practically constant in the axial direction, except in the immediate vicinity of the roll ends. A corresponding behaviour in the radial pressure of a magnetic tape pack with the applied winding tension linearly 
distributed across the tape pack width is found in [4], although no notice was taken of the phenomenon. A closer examination reveals a minor increase (3\%) in the radial pressure near the roll ends, while a much stronger edge effect is seen in the circumferential stress. Compressive circumferential stresses exceeding $2000 \mathrm{kPa}$ at the left end and $4000 \mathrm{kPa}$ at the right end are found (see Fig. 9 (b)). The axial and shear stress distributions for the finished roll are shown in Fig. 10. Comparison of Figs. 10 and 8 reveals an amazing similarity of the corresponding stress distributions. The axial stress distributions of Figs. 8 (a) and 10 (a) are almost identical. The shear stress distributions of Figs. 8 (b) and 10 (b) are also almost identical except that the values of the latter are shifted approximately $40 \mathrm{kPa}$ upwards. This shift increases the absolute values of the shear stress gradients near the right end of the roll. Therefore, the edge effect of the circumferential stress is also stronger there (see Fig. 9 (b) and Eq. (50)). The fact that the stress distributions seem to be very insensitive to the axial change of the incoming web tension proposes that a balancing mechanism exists in the roll. In fact, it is easy to see that this stress leveling mechanism is maintained by the Poisson's ratios $\nu_{r z}$ and $\nu_{\theta z}$ of the web. For example, an increased radial pressure in the right part of the roll brings about an increased compressive axial stress across the roll, which in turn increases the radial pressure and circumferential stress on the left part of the roll. For the circumferential stress the mechanism works in a similar way. In other words, a kind of "hydrostatic" stress balancing mechanism exists in the orthotropic paper roll. This conclusion was confirmed by a calculation where the Poisson's ratios were set to zero. The results clearly indicated that in this case the radial and circumferential stress distributions increased monotonously for increasing $z$ (excluding the edge effects), and that the axial and shear stresses were almost zero for most part of the roll.

\section{Conclusions}

In this work a two-dimensional axially symmetric winding model accounting for large deformations, centrifugal forces, and general axisymmetric core geometry was developed. A nonlinear orthotropic constitutive law, fulfilling the requirements of material frame indifference and orthotropic material symmetry, was used. Large deformations were modelled using the total Lagrangian formulation, the second Piola-Kirchhoff stress tensor and the Green-Lagrange strain tensor. The resulting equations were solved using the finite element method. The model was applied to the winding of paper rolls. In the calculated examples, compressive radial strains of the order of $10 \%$ were found, emphasizing the importance of a large deformation analysis in paper winding. It should be noted that strains of this magnitude have a considerable effect on the length of the newly added layer and, therefore, on the roll mass. This must be taken into account, for example, in vibration models where the roll mass is expressed as a function of the number of layers in the roll. The model was used to study the effect of centrifugal forces during winding on the stresses of the finished nonrotating roll. Due to the high speeds of modern winders, the centrifugal forces had a significant effect on the radial and circumferential stresses in the roll. By applying an additional, properly selected, compensatory incoming tension to the web, the changes in the roll stresses due to rotation could be effectively eliminated. For nonzero Poisson's ratios significant edge effects in the roll stresses were found. In the calculated cases, the radial pressure increased typically $10 \mathrm{kPa}$ and the circumferential compression even $3 \mathrm{MPa}$ at the roll ends. These changes can be a source of buckling or spoking defects near the roll edges. It was shown that the edge effects of the circumferential stress, in particular, were in close connection with the development of high shear stresses near the roll ends. Due to Poisson coupling, axial and shear stresses of magnitude $100-300 \mathrm{kPa}$ in the bulk of the roll were found. Due to strain mismatch at the core, axial stresses up to $500 \mathrm{kPa}$ in the immediate 
vicinity of the roll core emerged. High shear stress gradients (up to $40 \mathrm{kPa} / \mathrm{mm}$ ) were found near the roll ends, and especially, near the core. It was pointed out that these gradients are strongly contributing to the appearance of core bursts, a class of severe roll defects. It is evident that by properly selecting the elastic moduli of the core, the high stresses near the core could be at least partially eliminated. A novel stress leveling phenomenon in the roll was discovered. Namely, for a linearly distributed incoming web tension across the web width, almost width-independent radial and circumferential stress distributions were found. This leveling off could be attributed to the Poisson's ratios of the web. In future work, the effect of width-wise variation of the elastic moduli and thickness of the web on the finished roll should be studied, since these variations occur in real paper webs frequently.

\section{APPENDIX I}

The values of the material parameters and dimensions used in the calculations.

\begin{tabular}{lcr}
\hline Roll & & \\
Radial modulus coefficient & $C_{0}$ & $3.518 \mathrm{MPa}$ \\
Radial modulus coefficient & $C_{1}$ & 12.815 \\
Elastic moduli & $E_{\theta}$ & $5100 \mathrm{MPa}$ \\
& $E_{z}$ & $2600 \mathrm{MPa}$ \\
Shear modulus & $G_{r z}$ & $26 \mathrm{MPa}$ \\
Poisson's ratios & $\nu_{\theta z}$ & 0.3 \\
& $\nu_{\theta r}$ & 1.71 \\
& $\nu_{z r}$ & 0.39 \\
\hline Core & & \\
Inner radius & $r_{i}$ & $44 \mathrm{~mm}$ \\
Outer radius & $r_{o}$ & $50 \mathrm{~mm}$ \\
Elastic moduli & $E_{r}$ & $130 \mathrm{MPa}$ \\
& $E_{\theta}$ & $6500 \mathrm{MPa}$ \\
Shear modulus & $E_{z}$ & $4000 \mathrm{MPa}$ \\
Poisson's ratios & $G_{r z}$ & $234 \mathrm{MPa}$ \\
& $\nu_{z \theta}$ & 0.3 \\
& $\nu_{\theta r}$ & 2.4 \\
& $\nu_{z r}$ & 3.0 \\
\hline
\end{tabular}

\section{REFERENCES}

[1] D. Roisum. Pushing web handling to the limit, Converting Magazine 17: 72-76 (1999).

[2] D. R. Roisum. The Mechanics of Winding, TAPPI Press, Atlanta USA (1994).

[3] N. Zabaras, S. Liu, J. Koppuzha and E. Donaldson. A hypoelastic model for computing the stresses in center-wound rolls of magnetic tape, ASME J. Appl. Mech. 61: 290-295 (1994).

[4] N. Zabaras and S. Liu. A theory for small deformation analysis of growing bodies with an application to the winding of magnetic tape packs, Acta Mechanica 111: 95-110 (1995).

[5] Y. M. Lee and J. A. Wickert. Stress field in finite width axisymmetric wound rolls, ASME J. Appl. Mech. 69: 130-138 (2002). 
[6] Y. M. Lee and J. A. Wickert. Width-wise variation of magnetic tape pack stresses, ASME J. Appl. Mech. 69: 358-369 (2002).

[7] H. C. Altmann. Formulas for computing the stresses in center-wound rolls, TAPPI Journal 51: 176-179 (1968).

[8] H. P. Yagoda. Resolution of a core problem in wound rolls, ASME J. Appl. Mech. 47: 847-854 (1980).

[9] D. Connolly and D. J. Winarski. Stress analysis of wound magnetic tape, ASLE Tribology and Mechanics of Magnetic Storage Media, Special Publication Vol:16. ASLE, 172-182 (1984).

[10] Z. Hakiel. Nonlinear model for wound roll stresses, TAPPI Journal 70: 113-117 (1987).

[11] M. S. Willet and W. L. Poesch. Determining the stress distributions in wound reels of magnetic tape using a nonlinear finite-difference approach, ASME J. Appl. Mech. 55: 365-371 (1988).

[12] R. C. Benson. A nonlinear wound roll model allowing for large deformation, ASME J. Appl. Mech. 62: 853-859 (1995).

[13] H. Tramposch. Relaxation of internal forces in a wound reel of magnetic tape, ASME J. Appl. Mech. 32: 865-873 (1965).

[14] H. Tramposch. Anisotropic relaxation of internal forces in a wound reel of magnetic tape, ASME J. Appl. Mech. 34: 888-894 (1967).

[15] J. C. Heinrich, D. Connolly and B. Bhushan. Axisymmetric, finite element analysis of stress relaxation in wound magnetic tapes, ASLE Trans. 29: 75-84 (1986).

[16] W. R. Qualls and J. K. Good. An orthotropic viscoelastic winding model including a nonlinear radial stiffness, ASME J. Appl. Mech. 64: 201-208 (1997).

[17] S. Li and J. Cao. A hybrid approach for quantifying the winding process and material effects on sheet coil deformation, ASME J. Eng. Mater. Tech. 126: 303-313 (2004).

[18] J. E. Olsen. On the effect of centrifugal force on winding, TAPPI Journal 78: 191-195 (1995).

[19] J.K. Good, J.D. Pfeiffer and R.M. Giachetto. Losses in wound-on tension in the centerwinding of wound rolls, Web Handling, ASME AMD-149, ASME, New York, 1-12 (1992).

[20] A. J. M. Spencer. The formulation of constitutive equation for anisotropic solids, In: J.-P. Boehler (ed.) Mechanical behaviour of anisotropic solids, Proceedings of the Euromech Colloquium 115, June 1979, 19-22. Martinus Nijhoff publishers, Hague, Netherlands, 2-26 (1982).

[21] J. D. Pfeiffer. Measurement of the K2 factor for paper, Tappi Journal 64: 105-106 (1981).

[22] M. Ilomäki. Application of fracture mechanics in analyzing delemination of cyclically loaded paperboard core, Doctoral thesis, University of Oulu, Finland (2004). 
[23] D. B. Bogy. On the problem of edge-bonded elastic quarter-planes loaded at the boundary, Int. J. Solids Struct. 6: 1287-1313 (1970).

[24] E. S. Folias. On the stress singularities at the intersection of a cylindrical inclusion with the free surface of a plate, Int. J. Frac. 39: 25-34 (1989).

[25] S. S. Wang and I. Choi. Boundary-Layer Effects in Composite Laminates: Part 1Free-Edge Stress Singularities, ASME J. Appl. Mech. 49: 541-548 (1982). 